\title{
Finite-time Synchronization of Large-scale Hyper Chaotic Systems with Parametric Uncertainties and Time-varying Delay
}

\author{
Qiaoping $\mathrm{Li}^{\mathrm{a}} \mathrm{b}, *$, Sanyang $\operatorname{Liu}^{\mathrm{a}}$, Li Ge $\mathrm{e}^{\mathrm{a}, \mathrm{b}}$ \\ a School of Mathematics and Statistics, Xidian University, Xi'an 710071, China \\ b School of Mathematical Sciences, Henan Institute of Science and Technology, Xinxiang 453003 China
}

*Corresponding author, e-mail: liqiaoping1981@126.com

Received 14 May 2018

Accepted 2 Aug 2018

\begin{abstract}
In this paper, the finite-time synchronization of large-scale hyper chaotic systems with parametric uncertainties and time-varying delay is investigated. Based on Lyapunov stability theory and finite-time control technique, a suitable time-delay state feedback controller is designed, and a sufficient condition of finite-time synchronization is put forward. Finally, the numerical simulation is presented to illustrate the applicability and effectiveness of the proposed method.
\end{abstract}

KEYWORDS: finite-time control technique; combined chaotic system; uncertain parameter; nonlinear control

MSC2010: 93C10, 93C85

\section{INTRODUCTION}

Due to its potential applications for secure communication, engineering science, and other fields, the synchronization of chaotic systems has been an active research object ${ }^{1}$. However, most of the previous researches were primarily concerned with the asymptotic stability of the synchronization error system $^{2}$. In reality, optimizing the synchronization time is more valuable than achieving asymptotic synchronization. To realize fast synchronization, the exponential synchronization which can ensure the response system converge to the drive system at an exponential rate has been proposed ${ }^{3}$. Later, the finite-time control technique arises naturally in time-optimal control ${ }^{4}$. Its key feature is to drive the controlled system to its target within a finite time and to keep them there then after ${ }^{5}$.

In many practical applications, the influence of parametric uncertainties can not be ignored, otherwise, the stability of the synchronization error system would be destroyed ${ }^{6}$. The problem of finitetime synchronization between two chaotic systems with unknown parameters has been addressed ${ }^{7}$, however, the time-delay has not been considered. In fact, time-delays exist widely in reality because of finite signal transformation times and memory effects. For delayed chaotic system, the relationship between the drive and the response systems are more complex. The finite-time synchronization of chaotic systems with time-delay has been studied ${ }^{8}$, but the parametric uncertainty has not been taken into account, and what is more, it only considers the simple case in which the time-delay is constant. It is concluded from the recent research that timedelayed system is still vulnerable for communication because the time delay $\tau$ can be exposed by several measures, such as filling factor ${ }^{9}$, autocorrelation ${ }^{10}$, one step prediction error ${ }^{11}$ and so on. If the delay time $\tau$ is known, the time delayed system becomes quite a simple one, and the message encoded by the chaotic signal can be extracted by the common attack method ${ }^{12}$. Therefore, to realize the finite-time synchronization of chaotic systems with parametric uncertainties and time-varying delay, the aforementioned methods are no longer suitable, and a new analytical control scheme should be presented.

Furthermore, it is worth pointing out that all the synchronization methods mentioned above are involved in one drive and one response system. However, in practice, since the configuration of synchronization is limited to a single pair of one way coupled oscillations, this method can not be applied for multiuser communication systems ${ }^{13}$. Since it can further improve the security when the synchronization technique is applied in the secure communication, the technique of multiplexing is 
therefore a very important issue for high-capacity communications. In addition, the level of the communication security depends on the complexity level of the drive dynamical system and signal loading scheme. If the driving system involves more chaotic subsystems and the way of combination among subsystems is more complex, then the geometry topological manifold of the compound driving system will become more complex, and the transmitted signal will be separated into several parts carried by different subsystems to improve the anti-attack ability and anti-translated capability. R. Luo proposed the combination synchronization which contains two drive systems and one response system ${ }^{14}$. Recently, the dual synchronization were investigated which involves two drive systems and two response systems ${ }^{15}$. Combining the advantages of the two methods mentioned above, dual combination synchronization which involves four drive systems and two response systems has been put forward ${ }^{16}$. However, the disadvantage of these combination methods is that the subsystems involved must have the same dimension and the dimension of the combined system does not change. In fact, the higher the dimension of chaotic system, the more complex of the chaotic topology. This inspires our work.

By combining $k$ ordinary chaotic systems into one chaotic system (the dimensions of the subsystems can be the same or different), we can obtain a new drive system. Because the compound system involves multiple subsystems and its dimension increases significantly, we call it large-scale hyper chaotic system. Using the same method, we construct another large-scale hyper chaotic system by a group of ordinary chaotic systems as the response system. It is obvious that the problem of the synchronization between these two large-scale hyper chaotic systems are more general and of more practical significance.

Inspired by the discussion above, in this paper, we deal with the finite-time synchronization of large-scale hyper chaotic systems with parametric uncertainties and time-varying delay. On the basic of the finite-time control technique, a nonlinear controller is designed. Furthermore, a sufficient condition of finite-time synchronization in the form of linear matrix inequalities (LMIs) is established. Finally, the numerical simulation is put forward to demonstrate the feasibility and correctness of the advanced scheme.

Compared with the existing literatures, there are two advantages which make the proposed com- munication scheme attractive. Firstly, both the drive system and the response system are the compound of multiple chaotic subsystems. In the process of secret communication, the transmitted signals can be split into several different parts and loaded in different subsystems to improve the security of signal transmission. In addition, the dimension of the compound system increases significantly, which lead to the chaotic topological manifold becoming more complex. Secondly, by adding a nonlinear term to the traditional linear controller, fast synchronization is effectively achieved.

\section{PROBLEM DESCRIPTION}

Taking into account the parametric uncertainties and time-varying delay, we consider a group of chaotic systems as the master dynamics, which is described by

$$
\begin{aligned}
\dot{x}^{i}(t)= & \left(A^{i}+\Delta A^{i}\right) x^{i}(t)+\left(B^{i}+\Delta B^{i}\right) x^{i}(t-\tau(t)) \\
& +\left(C^{i}+\Delta C^{i}\right) f^{i}\left(x^{i}(t)\right) \\
& +\left(D^{i}+\Delta D^{i}\right) f^{i}\left(x^{i}(t-\tau(t))\right) \quad(1 \leqslant i \leqslant k),
\end{aligned}
$$

where $x^{i}(t)=\left[x_{1}^{i}(t) \cdots x_{n}^{i}(t)\right]^{T} \in \mathbb{R}^{n}$ is the state vector of the $i$ th master system, and $f^{i}\left(x^{i}(\cdot)\right)=$ $\left[f_{1}^{i}\left(x^{i}(\cdot)\right) \cdots f_{n}^{i}\left(x^{i}(\cdot)\right)\right]^{T} \in \mathbb{R}^{n}$ is continuous nonlinear function, where $A^{i}, B^{i}, C^{i}$ and $D^{i}$ are known $n \times n$ constant matrices, $\Delta A^{i}, \Delta B^{i}, \Delta C^{i}$ and $\Delta D^{i}$ are parametric uncertainties, and $\tau(t)$ is the transmission time-varying delay. by

Correspondingly, the response system is given

$$
\begin{aligned}
\dot{y}^{i}(t)= & \left(A^{i}+\Delta \hat{A}^{i}\right) y^{i}(t)+\left(B^{i}+\Delta \hat{B}^{i}\right) y^{i}(t-\tau(t)) \\
+ & \left(C^{i}+\Delta \hat{C}^{i}\right) f^{i}\left(y^{i}(t)\right)+\left(D^{i}+\Delta \hat{D}^{i}\right) \\
& \times f^{i}\left(y^{i}(t-\tau(t))\right)+u^{i}(t) \quad(1 \leqslant i \leqslant k),
\end{aligned}
$$

where $y^{i}(t)=\left[y_{1}^{i}(t) \cdots y_{n}^{i}(t)\right]^{T} \in \mathbb{R}^{n}$ is the state vector of the $i$ th response system, $\Delta \hat{A}^{i}, \Delta \hat{B}^{i}, \Delta \hat{C}^{i}$ and $\Delta \hat{D}^{i}$ are parametric uncertainties, and $u(t)=$ $\left[u_{1}(t) \cdots u_{n}(t)\right]^{T} \in \mathbb{R}^{n}$ is the vector of control input.

For convenience, define

$$
\begin{aligned}
x(\cdot) & =\left[\left(x^{1}(\cdot)\right)^{T}, \ldots,\left(x^{k}(\cdot)\right)^{T}\right]^{T}, \\
y(\cdot) & =\left[\left(y^{1}(\cdot)\right)^{T}, \ldots,\left(y^{k}(\cdot)\right)^{T}\right]^{T}, \\
f(\cdot) & =\left[\left(f^{1}(\cdot)\right)^{T}, \ldots,\left(f^{k}(\cdot)\right)^{T}\right]^{T}, \\
u(\cdot) & =\left[\left(u^{1}(\cdot)\right)^{T}, \ldots,\left(u^{k}(\cdot)\right)^{T}\right]^{T}, \\
A & =\operatorname{diag}\left\{A^{1}, A^{2}, \ldots, A^{k}\right\},
\end{aligned}
$$




$$
\begin{aligned}
B & =\operatorname{diag}\left\{B^{1}, B^{2}, \ldots, B^{k}\right\}, \\
C & =\operatorname{diag}\left\{C^{1}, C^{2}, \ldots, C^{k}\right\}, \\
D & =\operatorname{diag}\left\{D^{1}, D^{2}, \ldots, D^{k}\right\}, \\
\Delta A & =\operatorname{diag}\left\{\Delta A^{1}, \ldots, \Delta A^{k}\right\}, \\
\Delta B & =\operatorname{diag}\left\{\Delta B^{1}, \cdots, \Delta B^{k}\right\}, \\
\Delta C & =\operatorname{diag}\left\{\Delta C^{1}, \ldots, \Delta C^{k}\right\}, \\
\Delta D & =\operatorname{diag}\left\{\Delta D^{1}, \ldots, \Delta D^{k}\right\}, \\
\Delta \tilde{A} & =\operatorname{diag}\left\{\Delta \tilde{A}^{1}, \ldots, \Delta \tilde{A}^{k}\right\}, \\
\Delta \tilde{B} & =\operatorname{diag}\left\{\Delta \tilde{B}^{1}, \ldots, \Delta \tilde{B}^{k}\right\}, \\
\Delta \tilde{C} & =\operatorname{diag}\left\{\Delta \tilde{C}^{1}, \ldots, \Delta \tilde{C}^{k}\right\}, \\
\Delta \tilde{D} & =\operatorname{diag}\left\{\Delta \tilde{D}^{1}, \ldots, \Delta \tilde{D}^{k}\right\},
\end{aligned}
$$

and

$$
\begin{aligned}
\bar{A} & =A+\Delta A, & \bar{B} & =B+\Delta B, \\
\bar{C} & =C+\Delta C, & \bar{D} & =D+\Delta D, \\
\Delta \tilde{A}^{i} & =\Delta A^{i}-\Delta \hat{A}^{i}, & \Delta \tilde{B}^{i} & =\Delta B^{i}-\Delta \hat{B}^{i}, \\
\Delta \tilde{C}^{i} & =\Delta C^{i}-\Delta \hat{C}^{i}, & \Delta \tilde{D}^{i} & =\Delta D^{i}-\Delta \hat{D}^{i} .
\end{aligned}
$$

Then, the systems (1) and (2) are reduced respectively in the following forms

$$
\begin{gathered}
\dot{x}(t)=\bar{A} x(t)+\bar{B} x(t-\tau(t))+\bar{C} f(x(t)) \\
+\bar{D} f(x(t-\tau(t))), \\
\dot{y}(t)=(\bar{A}-\Delta \tilde{A}) y(t)+(\bar{B}-\Delta \tilde{B}) y(t-\tau(t)) \\
+(\bar{C}-\Delta \tilde{C}) f(y(t)) \\
+(\bar{D}-\Delta \tilde{D}) f(y(t-\tau(t)))+u(t),
\end{gathered}
$$

where $x(\cdot), y(\cdot), f(\cdot), u(\cdot) \in \mathbb{R}^{\bar{n}}, \bar{A}, \bar{B}, \bar{C}, \bar{D}, \Delta \tilde{A}, \Delta \tilde{B}$, $\Delta \tilde{C}, \Delta \tilde{D} \in \mathbb{R}^{\bar{n} \times \bar{n}}$, and $\bar{n}=k n$.

In order to obtain the main result, some reasonable assumptions and necessary lemmas are introduced below.

Lemma 1 (Ref. 3) Let $\Lambda \in \mathbb{R}^{n \times n}$ is a positive symmetric matrix, then, for any real matrices $X_{1}, X_{2} \in$ $\mathbb{R}^{n \times m}$, the following inequality holds

$$
X_{1}^{T} X_{2}+X_{2}^{T} X_{1} \leqslant X_{1}^{T} \Lambda^{-1} X_{1}+X_{2}^{T} \Lambda X_{2} .
$$

Specially, choosing $\Lambda=\varepsilon I$, we obtain

$$
X_{1}^{T} X_{2}+X_{2}^{T} X_{1} \leqslant \varepsilon^{-1} X_{1}^{T} X_{1}+\varepsilon X_{2}^{T} X_{2},
$$

where $\varepsilon$ is a positive scalar.

Lemma 2 (Ref. 17) Assume $V(t)$ is a continuous and positive definite function which satisfies the following differential inequality

$$
\dot{V}(t) \leqslant-b_{1} V^{\rho}(t)-b_{2} V(t), t \geqslant t_{0}, V\left(t_{0}\right) \geqslant 0
$$

where $b_{1}>0, b_{2}>0$ and $0<c<1$ are constants. Then, when $V^{1-\rho}\left(t_{0}\right) \leqslant b_{1} / b_{2}$, the following results are true

$$
V(t) \begin{cases}\leqslant e^{b_{2}\left(t-t_{0}\right)}\left[V^{1-\rho}\left(t_{0}\right)+\frac{b_{1}}{b_{2}}\right. & \\ \left.-\frac{b_{1}}{b_{2}} e^{-b_{2}(1-\rho)\left(t-t_{0}\right)}\right]^{1 /(1-\rho)} & \text { if } t_{0} \leqslant t<T, \\ =0 & \text { if } t \geqslant T,\end{cases}
$$

with $T$ given by

$$
T=t_{0}+\frac{1}{b_{2}(1-\rho)} \ln \left(1+\frac{b_{2} V^{1-\rho}\left(t_{0}\right)}{b_{1}}\right) .
$$

Assumption 1. For the time-varying delay $\tau(t)$, there exist non-negative constants $h$ and $q$, i.e.

$$
0 \leqslant \tau(t) \leqslant h, \quad 0 \leqslant \dot{\tau}(t) \leqslant q<1 .
$$

Assumption 2. Parametric uncertainties $\Delta A, \Delta B$, $\Delta C, \Delta D, \Delta \hat{A}, \Delta \hat{B}, \Delta \hat{C}$ and $\Delta \hat{D}$ are bounded, and they can be decomposed as

$$
\begin{aligned}
\Delta A & =H_{1} F_{1}(t) E_{1}, & & \Delta B=H_{2} F_{2}(t) E_{2}, \\
\Delta C & =H_{3} F_{3}(t) E_{3}, & & \Delta D=H_{4} F_{4}(t) E_{4}, \\
\Delta \hat{A} & =H_{1} \hat{F}_{1}(t) E_{1}, & & \Delta \hat{B}=H_{2} \hat{F}_{2}(t) E_{2}, \\
\Delta \hat{C} & =H_{3} \hat{F}_{3}(t) E_{3}, & \Delta \hat{D} & =H_{4} \hat{F}_{4}(t) E_{4},
\end{aligned}
$$

where $H_{j}, E_{j}$ are known constant matrices with appropriate dimensions, $F_{j}(t)$ and $\hat{F}_{j}(t)$ are uncertain matrices satisfying

$$
F_{j}(t)^{T} F_{j}(t) \leqslant I, \quad \hat{F}_{j}(t)^{T} \hat{F}_{j}(t) \leqslant I, \quad j=1,2,3,4 .
$$

Assumption 3. The difference between the corresponding parametric uncertainties of systems (1) and (2) are norm-bounded, i.e.

$$
\begin{array}{ll}
\|\Delta \tilde{A}\| \leqslant \alpha_{1}, & \|\Delta \tilde{B}\| \leqslant \alpha_{2}, \\
\|\Delta \tilde{C}\| \leqslant \alpha_{3}, & \|\Delta \tilde{D}\| \leqslant \alpha_{4},
\end{array}
$$

where $\alpha_{j}>0(j=1,2,3,4)$ are known real constants, and $\|\cdot\|$ represents the 2-norm.

Assumption 4. The parametric uncertainties and the time-varying delay do not destroy the chaotic behavior of the drive and response systems.

Definition 1 For the large-scale drive system (3) and the large-scale response system (4), it is said that these two systems are complete synchronization, if

$$
\lim _{t \rightarrow \infty}\|x(t)-y(t)\|=0 .
$$


Definition 2 For the large-scale drive system (3) and the large-scale response system (4), if there exists a constant $T>0$, such that

$$
\left\{\begin{array}{l}
\lim _{t \rightarrow T}\|x(t)-y(t)\|=0, \\
\|x(t)-y(t)\|=0 \quad \text { if } t \geqslant T,
\end{array}\right.
$$

then it is said that these two large-scale hyper chaotic systems are finite-time synchronization.

Assumption 5. The nonlinear functions $f_{i}(\cdot)$ are bounded, and satisfy the Lipschitz condition, namely there exist positive constants $l_{1}, \cdots, l_{\bar{n}}$ such that

$$
\left|f_{i}(a)-f_{i}(b)\right| \leqslant l_{i}|a-b|, \quad i=1,2, \cdots, \bar{n} .
$$

Denote $l=\max \left\{l_{1}, \cdots, l_{\bar{n}}\right\}$, it follows that

$$
\|f(x(\cdot))-f(y(\cdot))\| \leqslant l\|x(\cdot)-y(\cdot)\|=l\|e(\cdot)\| .
$$

The main object in this paper is to design an appropriate controller $u(t)$ to guarantee the largescale hyper chaotic systems (3) and (4) are finitetime synchronization.

We define the synchronization error vector

$$
e(t)=x(t)-y(t) .
$$

Combining systems (3),(4) with the error vector (7), the following error dynamical system is obtained

$$
\begin{aligned}
\dot{e}(t)= & \dot{x}(t)-\dot{y}(t) \\
= & \bar{A} x(t)+\bar{B} x(t-\tau(t))+\bar{C} f(x(t)) \\
& +\bar{D} f(x(t-\tau(t)))-[(\bar{A}-\Delta \tilde{A}) y(t) \\
& +(\bar{B}-\Delta \tilde{B}) y(t-\tau(t))+(\bar{C}-\Delta \tilde{C}) f(y(t)) \\
& +(\bar{D}-\Delta \tilde{D}) f(y(t-\tau(t)))+u(t)],
\end{aligned}
$$

that is

$$
\begin{aligned}
\dot{e}(t)= & \bar{A} e(t)+\bar{B} e(t-\tau(t))+\bar{C}[f(x(t)) \\
& -f(y(t))]+\bar{D}[f(x(t-\tau(t))) \\
- & f(y(t-\tau(t)))]+\Delta \tilde{A} y(t) \\
& +\Delta \tilde{B} y(t-\tau(t))+\Delta \tilde{C} f(y(t)) \\
+ & \Delta \tilde{D} f(y(t-\tau(t)))-u(t),
\end{aligned}
$$

based on what will be proposed in the main results.

\section{MAIN RESULTS}

It is clear that the finite-time synchronization of these two large-scale hyper chaotic systems is directly equivalent to the finite-time stabilization of the error system (8). In this section, we will pay our attention to design a suitable feedback controller to ensure the error trajectory converges to zero within a finite time. Without loss of generality, we employ the time-delay state feedback controller written by

$$
\begin{aligned}
u(t)= & K_{1} e(t)+K_{2} e(t-\tau(t)) \\
+ & \operatorname{sgn} e(t)\left[\alpha_{1} Y(t)+\alpha_{2} Y(t-\tau(t))\right. \\
& \left.\quad+\alpha_{3} F(y(t))+\alpha_{4} F(y(t-\tau(t)))\right] \\
+ & \frac{\gamma}{2}\left[e^{T}(t) P e(t)\right. \\
& \left.+\int_{t-\tau(t)}^{t} e^{T}(s) Q e^{-\beta(t-s)} e(s) d s\right]^{\eta} \frac{P e(t)}{\|P e(t)\|^{2}},
\end{aligned}
$$

with

$$
\left\{\begin{array}{c}
Y(t)=\left[\left|y_{1}(t)\right|, \cdots,\left|y_{\bar{n}}(t)\right|\right]^{T}, \\
Y(t-\tau(t))=\left[\left|y_{1}(t-\tau(t))\right|,\right. \\
\left.\ldots,\left|y_{\bar{n}}(t-\tau(t))\right|\right]^{T}, \\
F(y(t))=\left[\left|f_{1}(y(t))\right|, \cdots,\left|f_{\bar{n}}(y(t))\right|\right]^{T}, \\
F(y(t-\tau(t)))=\left[\left|f_{1}(y(t-\tau(t)))\right|,\right. \\
\left.\quad \ldots,\left|f_{\bar{n}}(y(t-\tau(t)))\right|\right]^{T}, \\
\operatorname{sgn} e(t)=\operatorname{diag}\left\{\operatorname{sgn} e_{1}(t), \cdots, \operatorname{sgn} e_{\bar{n}}(t)\right\},
\end{array}\right.
$$

where $K_{1}, K_{2} P$ and $Q$ are control gain matrices with appropriate dimensions, $\gamma>0$ and $0<\eta<1$ are known constants.

Remark 1 According to Eqs. (9), the control input $u(t)$ contains the factor $P e(t) /\|P e(t)\|^{2}$. In fact, when the error trajectory $e(t)=0$, it is obvious that $\|P e(t)\|^{2}=0$, which means $P e(t) /\|P e(t)\|^{2}$ is singular. In order to overcome this disadvantage, the control law (9) is modified as follows

$$
\begin{aligned}
u(t)=K_{1} e(t) & +K_{2} e(t-\tau(t)) \\
& +\operatorname{sgn} e(t)\left[\alpha_{1} Y(t)+\alpha_{2} Y(t-\tau(t))\right. \\
& \left.+\alpha_{3} F(y(t))+\alpha_{4} F(y(t-\tau(t)))\right] \\
+ & \frac{\gamma}{2}\left[e^{T}(t) P e(t)\right. \\
& \left.+\int_{t-\tau(t)}^{t} e^{T}(s) Q e^{-\beta(t-s)} e(s) d s\right]^{\eta} \Delta
\end{aligned}
$$

with

$$
\Delta= \begin{cases}\frac{P e(t)}{\|P e(t)\|^{2}} & \text { if }\|P e(t)\| \geqslant \delta, \\ 0 & \text { if }\|P e(t)\|<\delta,\end{cases}
$$

where the switching gain $\delta$ is a sufficiently small positive constant which can be chosen according to the designer's requirement. 
Remark 2 Another effective approach is using the function $P e(t) /\left(\|P e(t)\|^{2}+\xi\right)$ to approximate $P e(t) /\|P e(t)\|^{2}$, where $\xi$ is a small positive constant. Then the control law (9) is modified as

$$
\begin{aligned}
u(t)= & K_{1} e(t)+K_{2} e(t-\tau(t)) \\
& +\operatorname{sgn} e(t)\left[\alpha_{1} Y(t)+\alpha_{2} Y(t-\tau(t))\right. \\
& \left.\quad+\alpha_{3} F(y(t))+\alpha_{4} F(y(t-\tau(t)))\right] \\
& +\frac{\gamma}{2}\left[e^{T}(t) P e(t)\right. \\
+ & \left.\int_{t-\tau(t)}^{t} e^{T}(s) Q e^{-\beta(t-s)} e(s) d s\right]^{\eta} \frac{P e(t)}{\|P e(t)\|^{2}+\xi} .
\end{aligned}
$$

Based on the above controller, a sufficient condition of the finite-time synchronization in terms of LMIs is established as follows.

Theorem 1 If there exist symmetrical positive matrices $P, Q, R, T$, positive diagonal matrices $M, N$, control gain matrices $K_{1}, K_{2}$ with appropriate dimensions, and positive real scalars $\varepsilon_{1}, \varepsilon_{2}, \varepsilon_{3}, \varepsilon_{4}$, such that the following LMI holds

$$
\tilde{\Omega}=\left[\begin{array}{ccccc}
\tilde{\Omega}_{1} & \tilde{\Omega}_{2} & \tilde{\Omega}_{3} & \tilde{\Omega}_{4} & \tilde{\Omega}_{5} \\
* & -\varepsilon_{1} I & 0 & 0 & 0 \\
* & * & -\varepsilon_{2} I & 0 & 0 \\
* & * & * & -\varepsilon_{3} I & 0 \\
* & * & * & * & -\varepsilon_{4} I
\end{array}\right]<0
$$

where

$$
\begin{aligned}
\tilde{\Omega}_{1} & =\left[\begin{array}{cccc}
\tilde{\Omega}_{11} & \tilde{\Omega}_{12} & P C & P D \\
* & \tilde{\Omega}_{22} & 0 & 0 \\
* & * & \tilde{\Omega}_{33} & 0 \\
* & * & * & \tilde{\Omega}_{44}
\end{array}\right] \\
\tilde{\Omega}_{2} & =\left[H_{1}^{T} P, 0,0,0\right]^{T}, \\
\tilde{\Omega}_{3} & =\left[H_{2}^{T} P, 0,0,0\right]^{T}, \\
\tilde{\Omega}_{4} & =\left[H_{3}^{T} P, 0,0,0\right]^{T}, \\
\tilde{\Omega}_{5} & =\left[H_{4}^{T} P, 0,0,0\right]^{T},
\end{aligned}
$$

and

$$
\left\{\begin{array}{l}
\tilde{\Omega}_{11}=P A+A^{T} P-2 R+Q+l^{2} M \\
\quad+\beta P+\varepsilon_{1} E_{1}^{T} E_{1}, \\
\tilde{\Omega}_{12}=P B-T, \quad \\
\tilde{\Omega}_{22}=l^{2} N-\eta e^{-\beta h} Q+\varepsilon_{2} E_{2}{ }^{T} E_{2}, \\
\tilde{\Omega}_{33}=-M+\varepsilon_{3} E_{3}{ }^{T} E_{3}, \\
\tilde{\Omega}_{44}=-N+\varepsilon_{4} E_{4}{ }^{T} E_{4}, \\
R=P K_{1}, \\
T=P K_{2}, \\
\eta=1-q,
\end{array}\right.
$$

here $*$ denotes the symmetrical block in one symmetrical matrix.

Then the large-scale hyper chaotic systems (3) and (4) are finite-time synchronization under the feedback controller (9).

Proof: Choose the following Lyapunov-Krasovskii type function

$$
V(t)=V_{1}(t)+V_{2}(t)
$$

with

$$
\begin{aligned}
& V_{1}(t)=e^{T}(t) P e(t), \\
& V_{2}(t)=\int_{t-\tau(t)}^{t} e^{T}(s) Q e^{-\beta(t-s)} e(s) d s .
\end{aligned}
$$

Differentiating $V_{1}(t)$ along the error system, we obtain

$$
\begin{aligned}
\dot{V}_{1}(t)= & 2 e^{T} P \dot{e} \\
= & 2 e^{T} P\{\bar{A} e(t)+\bar{B} e(t-\tau(t))+\bar{C}[f(x(t)) \\
& \quad-f(y(t))]+\bar{D}[f(x(t-\tau(t))) \\
- & f(y(t-\tau(t)))]+\Delta \tilde{A} y(t)+\Delta \tilde{B} y(t-\tau(t)) \\
& +\Delta \tilde{C} f(y(t))+\Delta \tilde{D} f(y(t-\tau(t)))-u(t)\} .
\end{aligned}
$$

Submitting (9) into (8) and using the fact

$$
e^{T}(t) P \frac{P e(t)}{\|P e(t)\|^{2}}=1,
$$

we get

$$
\begin{aligned}
& \dot{V}_{1}(t)= 2 e^{T}(t) P\left(\bar{A}-K_{1}\right) e(t)+2 e^{T}(t) P\left(\bar{B}-K_{2}\right) \\
& \times e(t-\tau(t))+2 e^{T} P \bar{C}[f(x(t))-f(y(t))] \\
&+ 2 e^{T}(t) P \bar{D}[f(x(t-\tau(t))) \\
&-f(y(t-\tau(t)))]+2 e^{T}(t) P \\
& \times {[\Delta \tilde{A} y(t)+\Delta \tilde{B} y(t-\tau(t))+\Delta \tilde{C}} \\
&f(y(t))+\Delta \tilde{D} f(y(t-\tau(t)))] \\
&- 2 e^{T}(t) P \operatorname{sgn} e(t)\left[\alpha_{1} Y(t)+\alpha_{2} Y(t-\tau(t))\right. \\
&+\left.\alpha_{3} F(y(t))+\alpha_{4} F(y(t-\tau(t)))\right] \\
&-\gamma V^{\eta}(t) .
\end{aligned}
$$

Using Assumption 3 and the fact

$$
\begin{aligned}
& y(t) \leqslant Y(t), \quad f(y(t)) \leqslant F(y(t)), \\
& y(t-\tau(t)) \leqslant Y(t-\tau(t)), \\
& f(y(t-\tau(t))) \leqslant F(y(t-\tau(t))), \\
& Y(t) \geqslant 0, \quad Y(t-\tau(t)) \geqslant 0, \\
& F(y(t)) \geqslant 0, \quad F(y(t-\tau(t))) \geqslant 0,
\end{aligned}
$$




$$
e^{T}(t) \operatorname{sgn} e(t)=\left[\left|e_{1}(t)\right|, \ldots,\left|e_{\bar{n}}(t)\right|\right]^{T},
$$

we obtain the following inequations

$$
\begin{aligned}
& e^{T}(t) P \Delta \tilde{A} y(t) \leqslant \alpha_{1} e^{T}(t) \operatorname{sgn} e(t) P Y(t), \\
& e^{T}(t) P \Delta \tilde{B} y(t-\tau(t)) \\
& \quad \leqslant \alpha_{2} e^{T}(t) \operatorname{sgn} e(t) P Y(t-\tau(t)), \\
& e^{T}(t) P \Delta \tilde{C} f(y(t)) \leqslant \alpha_{3} e^{T}(t) \operatorname{sgn} e(t) P F(y(t)), \\
& e^{T}(t) P \Delta \tilde{D} f(y(t-\tau(t))) \\
& \quad \leqslant \alpha_{4} e^{T}(t) \operatorname{sgn} e(t) P F(y(t-\tau(t))),
\end{aligned}
$$

which yields

$$
\begin{aligned}
\dot{V}_{1}(t) \leqslant & 2 e^{T}(t) P\left(\bar{A}-K_{1}\right) e(t) \\
+ & 2 e^{T}(t) P\left(\bar{B}-K_{2}\right) e(t-\tau(t)) \\
& +2 e^{T}(t) P \bar{C}[f(x(t))-f(y(t))] \\
+ & 2 e^{T}(t) P \bar{D}[f(x(t-\tau(t))) \\
& \quad-f(y(t-\tau(t)))]-\gamma V^{\eta}(t) .
\end{aligned}
$$

Applying Lemma 1, we obtain the following relationships

$$
\begin{gathered}
2 e^{T}(t) P \bar{C}[f(x(t))-f(y(t))] \\
\leqslant e^{T}(t) P \bar{C} M^{-1} \bar{C}^{T} P e(t)+[f(x(t)) \\
-f(y(t))]^{T} M[f(x(t))-f(y(t))], \\
2 e^{T}(t) P \bar{D}[f(x(t-\tau(t)))-f(y(t-\tau(t)))] \\
\leqslant e^{T}(t) P \bar{D} N^{-1} \bar{D}^{T} P e(t)+[f(x(t-\tau(t))) \\
-f(y(t-\tau(t)))]^{T} N[f(x(t-\tau(t))) \\
-f(y(t-\tau(t)))] .
\end{gathered}
$$

where $M$ and $N$ are positive diagonal matrices. So we have

$$
\begin{aligned}
& \dot{V}_{1}(t) \leqslant e^{T}(t)\left[P\left(\bar{A}-K_{1}\right)+\left(\bar{A}-K_{1}\right)^{T} P\right] e(t) \\
&+ 2 e^{T}(t) P\left(\bar{B}-K_{2}\right) e(t-\tau(t)) \\
&+e^{T}(t) P \bar{C} M^{-1} \bar{C}^{T} P e(t)+[f(x(t)) \\
&-f(y(t))]^{T} M[f(x(t))-f(y(t))] e^{T}(t) P \\
&+ \bar{D} N^{-1} \bar{D}^{T} P e(t) \\
&+ {[f(x(t-\tau(t)))-f(y(t-\tau(t)))]^{T} } \\
& \times N[f(x(t-\tau(t)))-f(y(t-\tau(t)))] \\
&-\gamma V^{\eta}(t) .
\end{aligned}
$$

Applying Assumption 5, we obtain

$$
\begin{aligned}
& {[f(x(t))-f(y(t))]^{T} M[f(x(t))-f(y(t))]} \\
& \quad \leqslant l^{2} e^{T}(t) M e(t)
\end{aligned}
$$

$$
\begin{aligned}
& {[f(x(t-\tau(t)))}-f(y(t-\tau(t)))]^{T} N \\
& \times[f(x(t-\tau(t)))-f(y(t-\tau(t)))] \\
& \leqslant l^{2} e^{T}(t-\tau(t)) N e(t-\tau(t)) .
\end{aligned}
$$

Then, (20) is reduced to

$$
\begin{aligned}
\dot{V}_{1}(t) \leqslant & e^{T}(t)\left[P\left(\bar{A}-K_{1}\right)+\left(\bar{A}-K_{1}\right)^{T} P\right] e(t) \\
& +2 e^{T}(t) P\left(\bar{B}-K_{2}\right) e(t-\tau(t)) \\
& +e^{T}(t) P \bar{C} M^{-1} \bar{C}^{T} P e(t)+e^{T}(t) P \\
& \times \bar{D} N^{-1} \bar{D}^{T} P e(t)+l^{2} e^{T}(t) M e(t) \\
& +l^{2} e^{T}(t-\tau(t)) N e(t-\tau(t))-\gamma V^{\eta}(t) .
\end{aligned}
$$

This is equivalent to

$$
\begin{aligned}
\dot{V}_{1}(t) \leqslant & e^{T}(t)\left[P\left(\bar{A}-K_{1}\right)+\left(\bar{A}-K_{1}\right)^{T} P\right. \\
+ & P \bar{C} M^{-1} \bar{C}^{T} P+P \bar{D} N^{-1} \bar{D}^{T} P \\
+ & \left.l^{2} M+\beta P\right] e(t)+e^{T}(t-\tau(t)) \\
& \times\left(l^{2} N\right) e(t-\tau(t))+2 e^{T}(t) P\left(\bar{B}-K_{2}\right) \\
& \times e(t-\tau(t))-\beta V_{1}(t)-\gamma V^{\eta}(t) . \quad(21)
\end{aligned}
$$

Notice $V_{2}(t)$ can be rewritten as

$$
V_{2}(t)=e^{-\beta t} \int_{t-\tau(t)}^{t} e^{T}(s) Q e^{\beta s} e(s) d s,
$$

the time derivative of $V_{2}(t)$ along the trajectory of the error system (8) is obtained as follows

$$
\begin{aligned}
\dot{V}_{2}(t)=-\beta e^{-\beta t} \int_{t-\tau(t)}^{t} e^{T}(s) Q e^{\beta s} e(s) d s \\
+e^{-\beta t} e^{T}(t) Q e^{\beta t} e(t) \\
-(1-\dot{\tau}(t)) e^{-\beta t} e^{T}(t-\tau(t)) Q \\
\times e^{\beta(t-\tau(t))} e(t-\tau(t)) \\
=-\beta V_{2}(t)+e^{T}(t) Q e(t)-(1-\dot{\tau}(t)) \\
\times e^{T}(t-\tau(t)) Q e^{-\beta \tau(t)} e(t-\tau(t)) .
\end{aligned}
$$

Since

$$
0 \leqslant \tau(t) \leqslant h, \quad 0 \leqslant \dot{\tau}(t) \leqslant q, \quad \eta=1-q,
$$

we have

$$
-(1-\dot{\tau}(t)) \leqslant-\eta, \quad-e^{-\beta \tau(t)} \leqslant-e^{-\beta h} .
$$

Then it follows by

$$
-(1-\dot{\tau}(t)) e^{-\beta \tau(t)} \leqslant-\eta e^{-\beta h},
$$

which yields

$$
\begin{aligned}
\dot{V}_{2}(t) \leqslant- & \beta V_{2}(t)+e^{T}(t) Q e(t) \\
& -\eta e^{T}(t-\tau(t)) Q e^{-\beta h} e(t-\tau(t)) .
\end{aligned}
$$


Combining (21) and (23), we have

$$
\begin{aligned}
\dot{V}(t) \leqslant & e^{T}(t)\left[P\left(\bar{A}-K_{1}\right)+\left(\bar{A}-K_{1}\right)^{T} P\right. \\
& +P \bar{C} M^{-1} \bar{C}^{T} P+P \bar{D} N^{-1} \bar{D}^{T} P \\
& \left.+Q+l^{2} M+\beta P\right] e(t) \\
+ & e^{T}(t-\tau(t))\left(l^{2} N-\eta e^{-2 \beta h} Q\right) e(t-\tau(t)) \\
+ & 2 e^{T}(t) P\left(\bar{B}-K_{2}\right) e(t-\tau(t)) \\
& -\beta V(t)-\gamma V^{\eta}(t) .
\end{aligned}
$$

For simplicity, we denote

$$
\Phi=\left[\begin{array}{cc}
\Phi_{11} & \Phi_{12} \\
* & \Phi_{22}
\end{array}\right],
$$

with

$$
\begin{aligned}
\Phi_{11}= & P\left(\bar{A}-K_{1}\right)+\left(\bar{A}-K_{1}\right)^{T} P \\
& +P \bar{C} M^{-1} \bar{C}^{T} P+P \bar{D} N^{-1} \bar{D}^{T} P \\
& +Q+l^{2} M+\beta P, \\
\Phi_{12}= & P\left(\bar{B}-K_{2}\right), \\
\Phi_{22}= & l^{2} N-\eta e^{-\beta h} Q .
\end{aligned}
$$

Then (24) is reduced to

$$
\begin{aligned}
\dot{V}(t) \leqslant\left(\begin{array}{c}
e(t) \\
e(t-\tau(t))
\end{array}\right)^{T} \Phi & \left(\begin{array}{c}
e(t) \\
e(t-\tau(t))
\end{array}\right) \\
& -\beta V(t)-\gamma V^{\eta}(t) .
\end{aligned}
$$

It is obvious that, if $\Phi<0$ then $\dot{V}(t)<-\beta V(t)-$ $\gamma V^{\eta}(t)$.

Applying Shure complement, we obtain that $\Phi<0$ if and only if

$$
\bar{\Omega}=\left[\begin{array}{cccc}
\bar{\Omega}_{11} & \bar{\Omega}_{12} & P \bar{C} & P \bar{D} \\
* & \bar{\Omega}_{22} & 0 & 0 \\
* & * & -M & 0 \\
* & * & * & -N
\end{array}\right]<0,
$$

where

$$
\begin{aligned}
\bar{\Omega}_{11}= & P\left(\bar{A}-K_{1}\right)+\left(\bar{A}-K_{1}\right)^{T} P+Q+l^{2} M \\
& +\beta P, \\
\bar{\Omega}_{12}= & \Phi_{12}=P\left(\bar{B}-K_{2}\right), \\
\bar{\Omega}_{22}= & \Phi_{22}=l^{2} N-\eta e^{-\beta h} Q .
\end{aligned}
$$

Combined with $\left(F_{j}(t)\right)^{T} F_{j}(t) \leqslant I, \quad j=1,2,3,4$, based on the Lemma 1 and Shure complement, we obtain a sufficient condition of $\bar{\Omega}<0$ as follows

$$
\tilde{\Omega}=\left[\begin{array}{ccccc}
\tilde{\Omega}_{1} & \tilde{\Omega}_{2} & \tilde{\Omega}_{3} & \tilde{\Omega}_{4} & \tilde{\Omega}_{5} \\
* & -\varepsilon_{1} I & 0 & 0 & 0 \\
* & * & -\varepsilon_{2} I & 0 & 0 \\
* & * & * & -\varepsilon_{3} I & 0 \\
* & * & * & * & -\varepsilon_{4} I
\end{array}\right]
$$

where

$$
\begin{aligned}
\tilde{\Omega}_{1} & =\left[\begin{array}{cccc}
\tilde{\Omega}_{11} & \tilde{\Omega}_{12} & P C & P D \\
* & \tilde{\Omega}_{22} & 0 & 0 \\
* & * & \tilde{\Omega}_{33} & 0 \\
* & * & * & \tilde{\Omega}_{44}
\end{array}\right] \\
\tilde{\Omega}_{2} & =\left[H_{1}^{T} P, 0,0,0\right]^{T}, \\
\tilde{\Omega}_{3} & =\left[H_{2}^{T} P, 0,0,0\right]^{T}, \\
\tilde{\Omega}_{4} & =\left[H_{3}^{T} P, 0,0,0\right]^{T}, \\
\tilde{\Omega}_{5} & =\left[H_{4}^{T} P, 0,0,0\right]^{T},
\end{aligned}
$$

and

$$
\begin{aligned}
\tilde{\Omega}_{11} & =P\left(A-K_{1}\right)+\left(A-K_{1}\right)^{T} P+Q+l^{2} M \\
& +\beta P+\varepsilon_{1} E_{1}{ }^{T} E_{1},
\end{aligned}
$$

To obtain the LMI-based conditions, we set

$$
R=P K_{1}, \quad T=P K_{2},
$$

it is clear that if the matrix $P$ is invertible, the control gains $K_{1}, K_{2}$ can be calculated by

$$
K_{1}=P^{-1} R, \quad K_{2}=P^{-1} T .
$$

Based on the above discussion, we conclude that

$$
\begin{aligned}
\tilde{\Omega}<0 & \Rightarrow \bar{\Omega}<0 \\
& \Rightarrow \Phi<0 \\
& \Rightarrow \dot{V}(t)<-\beta V(t)-\gamma V^{\eta}(t) .
\end{aligned}
$$

Applying Lemma 2, the large-scale chaotic systems (3) and (4) are finite-time synchronization when the LMI (14) holds, and $T$ is given by

$$
T=\frac{1}{\beta(1-\eta)} \ln \left(1+\frac{\beta V^{1-\eta}(0)}{\gamma}\right) .
$$

Hence, the proof is completed.

\section{NUMERICAL SIMULATION}

In our simulation, Lorenz system and Chen system with time-varying delay are chosen as the drive systems, which is described by

$$
\begin{gathered}
\dot{x}(t)=(A+\Delta A) x(t)+(B+\Delta B) x(t-\tau(t)) \\
+(C+\Delta C) f(x(t)) \\
+(D+\Delta D) f(x(t-\tau(t))),
\end{gathered}
$$



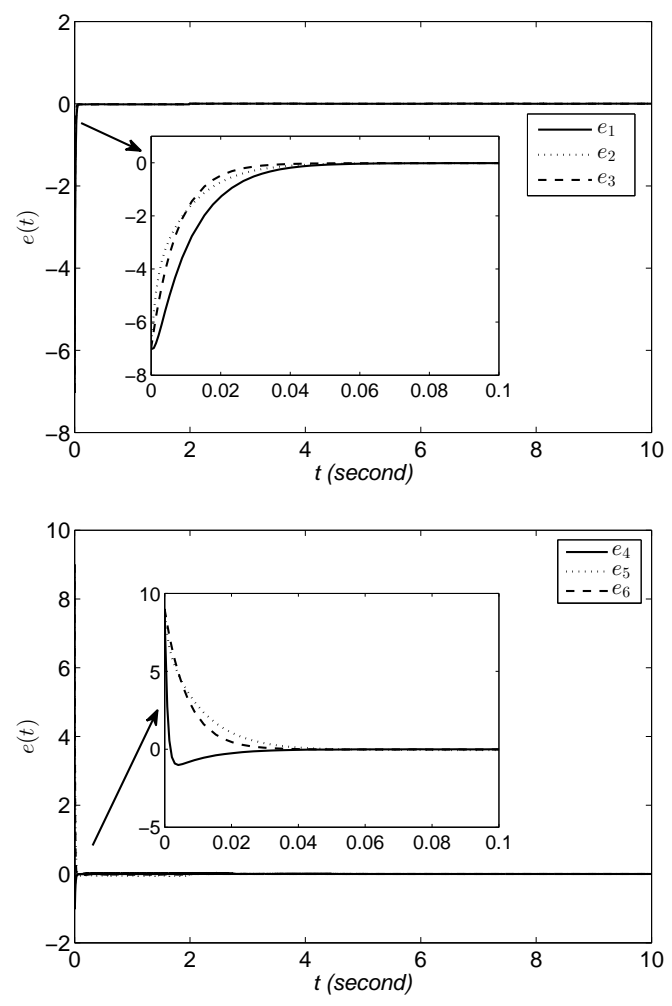

Fig. 1 Time response of the synchronization errors $e_{i}(t)$ with $\beta=\gamma=2$, (a) $i=1,2,3$, (b) $i=4,5,6$.

Meanwhile, the identical system with different parametric uncertainties and different initial value are considered as the response systems, which is described by

$$
\begin{aligned}
& \dot{y}(t)=(A+\Delta \hat{A}) y(t)+(B+\Delta \hat{B}) y(t-\tau(t)) \\
&+(C+\Delta \hat{C}) f(y(t)) \\
&+(D+\Delta \hat{D}) f(y(t-\tau(t)))+u(t),
\end{aligned}
$$

where

$$
\begin{aligned}
A & =\left[\begin{array}{cccccc}
-10 & 10 & 0 & 0 & 0 & 0 \\
28 & 1 & 0 & 0 & 0 & 0 \\
0 & 0 & -8 / 3 & 0 & 0 & 0 \\
0 & 0 & 0 & -35 & 35 & 0 \\
0 & 0 & 0 & -7 & 28 & 0 \\
0 & 0 & 0 & 0 & 0 & 3
\end{array}\right] \\
f(x(\cdot)) & =\left[0,-x_{1}(\cdot) x_{3}(\cdot), x_{1}(\cdot) x_{2}(\cdot), 0,\right. \\
& \left.-x_{4}(\cdot) x_{6}(\cdot), x_{4}(\cdot) x_{5}(\cdot)\right]^{T},
\end{aligned}
$$

and the parametric uncertainties are given as

$$
\begin{array}{r}
\Delta A=\operatorname{diag}\{0.1 \sin t,-0.1 \sin 3 t, 0.1 \sin t, \\
0.1 \cos 2 t, 0.1 \cos 4 t, 0.1 \sin t\},
\end{array}
$$
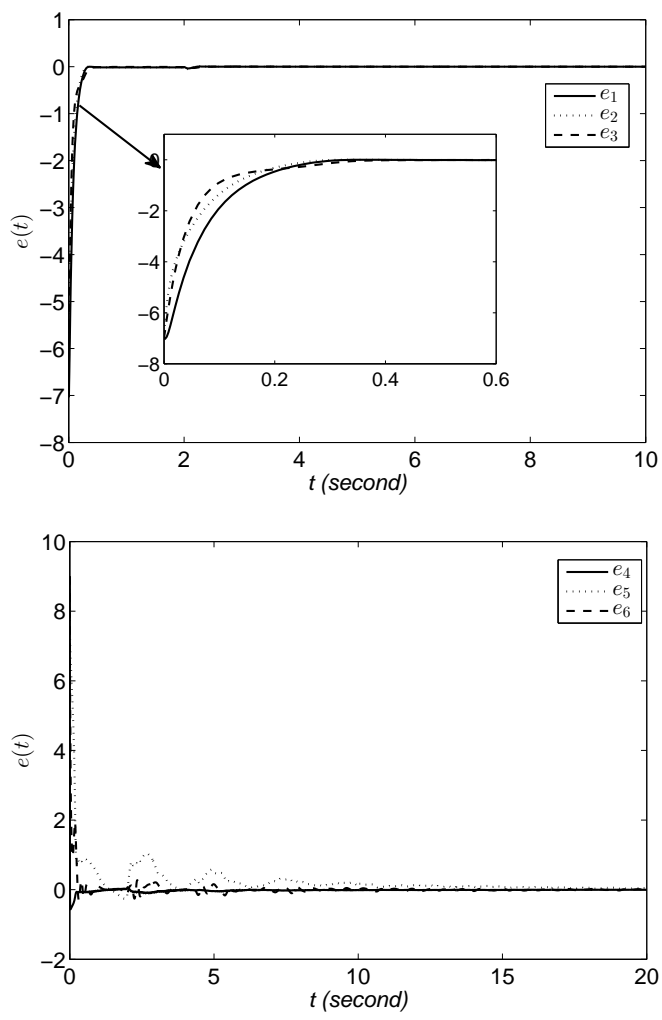

Fig. 2 Time response of the synchronization errors $e_{i}(t)$ with $\beta=\gamma=0$, (a) $i=1,2,3$, (b) $i=4,5,6$.

$$
\begin{gathered}
\Delta B=\operatorname{diag}\{0.01 \cos 2 t, 0.01 \sin t,-0.01 \\
\quad \sin 2 t, 0.01 \cos t, 0.01 \cos 2 t,-0.01 \cos t\}, \\
\Delta C=\operatorname{diag}\{-0.01 \cos 3 t,-0.01 \cos 3 t, 0.01 \sin t, \\
\quad-0.01 \cos 3 t, 0.01 \cos t, 0.01 \sin 3 t\}, \\
\Delta D=\operatorname{diag}\{0.04 \sin t,-0.04 \sin t, 0.04 \sin 3 t, \\
\quad 0.04 \cos t, 0.04 \sin t, 0.04 \cos 2 t\},
\end{gathered}
$$

and

$$
\begin{gathered}
\Delta \hat{A}=\operatorname{diag}\{-0.05 \sin t,-0.06 \sin 3 t,-0.03 \\
\cos 2 t, 0.08 \sin 2 t,-0.12 \sin 4 t, 0.2 \sin 2 t\}, \\
\Delta \hat{B}=\operatorname{diag}\{0.02 \cos t, 0.01 \sin 2 t, 0.01 \cos 2 t, \\
-0.02 \cos t, 0.01 \sin 2 t,-0.02 \cos t\}, \\
\Delta \hat{C}=\operatorname{diag}\{0.02 \sin 3 t,-0.01 \cos t, 0.02 \sin t, \\
-0.04 \cos 2 t, 0.01 \cos t,-0.01 \cos 2 t\}, \\
\Delta \hat{D}=\operatorname{diag}\{0.04 \sin t, 0.02 \cos 2 t, 0.04 \sin 2 t, \\
0.02 \sin 2 t, 0.04 \cos t,-0.01 \cos 2 t\},
\end{gathered}
$$

then, according to Assumption 2 we have

$$
H_{1}=0.1 I, H_{2}=0.1 I, H_{3}=0.1 I, H_{4}=0.2 I,
$$




$$
E_{1}=I, E_{2}=0.1 I, E_{3}=0.1 I, E_{4}=0.2 I .
$$

and

$$
\begin{gathered}
F_{1}(t)=\operatorname{diag}\{\sin t,-\sin 3 t, \sin t, \cos 2 t, \\
\cos 4 t, \sin t\}, \\
F_{2}(t)=\operatorname{diag}\{\cos 2 t, \sin t,-\sin 2 t, \cos t, \\
\cos 2 t,-\cos t\}, \\
F_{3}(t)=\operatorname{diag}\{-\cos 3 t,-\cos 3 t, \sin t,-\cos 3 t, \\
\cos t, \sin 3 t\}, \\
F_{4}(t)=\operatorname{diag}\{\sin t,-\sin t, \sin 3 t, \cos t, \\
\sin t, \cos 2 t\} .
\end{gathered}
$$

Choosing $\beta=2, \gamma=2, \eta=0.5$, using the MATLAB LMI Toolbox, we obtain

$$
K_{1}=\left[\begin{array}{cc}
K_{11} & 0 \\
0 & K_{12}
\end{array}\right], \quad K_{2}=\left[\begin{array}{cc}
K_{21} & 0 \\
0 & K_{22}
\end{array}\right],
$$

where

$$
\begin{aligned}
K_{11}= & {\left[\begin{array}{ccc}
152.1597 & -248.2540 & 0 \\
-229.9479 & 591.3069 & 0 \\
0 & 0 & 86.5369
\end{array}\right], } \\
K_{12}= & {\left[\begin{array}{ccc}
875.8940 & 214.3962 & 0 \\
151.3313 & 117.3855 & 0 \\
0 & 0 & 93.1439
\end{array}\right], } \\
K_{21}= & {\left[\begin{array}{ccc}
0.6639 & -1.0658 & 0 \\
-0.7290 & 2.7139 & 0 \\
0 & 0 & 0.3488
\end{array}\right], } \\
K_{22}= & {\left[\begin{array}{ccc}
3.0586 & 4.8151 & 0 \\
0.4773 & 3.0556 & 0 \\
0 & 0 & 0.3683
\end{array}\right] . }
\end{aligned}
$$

In the simulation, the drive system is started with $x(0)=(1,1,1,1,1,1)$ and the response system is initialized with $y(0)=(8,8,8,-8,-8,-8)$, the delay time $\tau(t)=2+\left(\cos ^{3} t\right) / 6$. Using the controller (13) with $\xi=0.01$, the synchronization error is revealed in Fig. 1. It is obvious that the synchronization error converges to zero in a short time.

When we choose the parameters $\beta=\gamma=0$, the controller (13) will reduce as an ordinary linear controller

$$
\begin{aligned}
u(t)=K_{1} e(t) & +K_{2} e(t-\tau(t)) \\
+ & \operatorname{sgn} e(t)\left[\alpha_{1} Y(t)+Y(t-\tau(t))\right. \\
& \left.+\alpha_{3} F(y(t))+\alpha_{4} F(y(t-\tau(t)))\right],
\end{aligned}
$$

then, the inequality

$$
\begin{aligned}
\dot{V}(t) \leqslant\left(\begin{array}{c}
e(t) \\
e(t-\tau(t))
\end{array}\right)^{T} \Phi & \left(\begin{array}{c}
e(t) \\
e(t-\tau(t))
\end{array}\right) \\
& -\beta V(t)-\gamma V^{\eta}(t)
\end{aligned}
$$

reduces to

$$
\dot{V}(t) \leqslant\left(\begin{array}{c}
e(t) \\
e(t-\tau(t))
\end{array}\right)^{T} \Phi\left(\begin{array}{c}
e(t) \\
e(t-\tau(t))
\end{array}\right) .
$$

Once the LMI (14) in Theorem 1 holds, we have $\Phi<0$, which yield $\dot{V}(t)<0$, applying Lyapunov stability theory, we derive the error system (8) is asymptotically stable. The simulation result is show in Fig. 2. In addition, the comparison between Fig. 1 and 2 shows that the control technique in this paper can effectively shorten the synchronization time, which verifies the superiority of the of the proposed method.

\section{CONCLUSION}

In this paper, we dealed with the finite-time synchronization of two large-scale hyper chaotic systems with parametric uncertainties and time-varying delay. Using the finite-time control technique and Lyapunov stability theory, we designed a suitable timedelay state feedback controller and put forward a sufficient finite-time synchronization condition in the form of LMI. The numerical simulation verified the correctness and effectiveness of the proposed scheme. In the further work, we will introduce the event-triggering mechanism based on the result of this paper to improve the ability of data filtering in the process of network synchronization and save the burden of the network.

Acknowledgements: This paper is supported by the National Natural Science Foundation of China (11702201), (61877046) and (61773156). thanks for all the references authors.

\section{REFERENCES}

1. Chen G, Dong X (1998) From chaos to order: methodologies, perspectives and applications. World Scientific Singapore 31, 113-22.

2. Lin J, Yan J (2009) Adaptive synchronization for two identical generalized Lorenz chaotic systems via a single controller. Nonlinear Anal RWA 10, 1151-9.

3. Zhang HG, Ma TD, Huang GB, Wang ZL (2010) Robust global exponential synchronization of uncertain chaotic delayed neural networks via dual-stage impulsive control. IEEE Trans syst man cybern Part $B$ 40, 831-43.

4. Kengne R, Tchitnga R, Mezatio A, Fomethe A, Litak G (2017) Finite-time synchronization of fractionalorder simplest two-component chaotic oscillators. Eur. Phys. J. B 90(88), 1-10. 
5. Chen X, Cao J, Park JH, Huang T, Qiu J (2018) Finitetime multi-switching synchronization behavior for multiple chaotic systems with network transmission mode. Journal of the Franklin Institute 355(5), 2892 911.

6. Lu J, Cao J (2005) Adaptive complete synchronization of two identical or different chaotic (hyperchaotic) systems with fully unknown parameters. Chaos, 15(4).

7. Aghababa MP, Khanmohammadi S, Alizadeh G (2011) Finite-time synchronization of two different chaotic systems with unknown parameters via sliding mode technique. Appl Math Model 35, 3080-91.

8. Mei J, Jiang MH, Xu WM, Wang B (2013) Finitetime synchronization control of complex dynamical networks with time delay. Commun Nonlinear Sci Numer Simulat 18, 2462-78.

9. Hegger R, Bunner MJ, Kantz H, Giaquinta A (1998) Identifying and modeling delay feedback systems. Phys Rev Lett 81, 558-61.

10. Arecchi FT, Meucci R, Allaria E, Di Garbo A (2002) Tsimring LS. Delayed self-synchronization in homoclinic chaos. Phys Rev E 65, 046237.

11. Ponomarenko VI, Prokhorov MD (2002) Extracting information masked by the chaotic signal of a timedelay system. Phys Rev E 66, 026215.

12. Ghosh D (2011) Projective-dual synchronization in delay dynamical systems with time-varying coupling delay. Nonlinear Dyn 66(4), 717-30.

13. Yoshimura K (1999) Multichannel digital communications by the synchronization of globally coupled chaotic systems. Phys Rev E 60, 1648-57.

14. Luo R, Wang Y, Deng S (2011) Combination synchronization of three classic chaotic systems using active backstepping design. Chaos 21, 043114.

15. Othman AA, Noorani MSM, Al-sawalha MM (2016) Adaptive dual synchronization of chaotic and hyperchaotic systems with fully uncertain parameters. Optik 127(19), 7852-64.

16. Sun J, Jiang S, Cui G, and Wang Y (2016) Dual combination synchronization of six chaotic systems. Journal of Bacteriology 14(1), 47-53.

17. Xu YH, Zhou WN, Fang JA, Xie CR, Tong DB (2016) Finite-time synchronization of the complex dynamical network with non-derivative and derivative coupling. Neurocomputing 173, 1356-61. [index heading! index subheading]:

finite-time control technique; combined chaotic system; uncertain parameter; nonlinear control 